\title{
Irritable bowel syndrome and inflammatory bowel disease overlap syndrome: pieces of the puzzle are falling into place
}

\author{
Rafiz Abdul Rani ${ }^{1}$, Raja Affendi Raja Ali ${ }^{2}$, Yeong Yeh Lee ${ }^{3}$ \\ ${ }^{I}$ Gastroenterology Unit, Faculty of Medicine, Universiti Teknologi MARA, Sungai Buloh, ${ }^{2}$ Gastroenterology Unit, Faculty of Medicine, Universiti \\ Kebangsaan Malaysia, Kuala Lumpur, ${ }^{3}$ School of Medical Sciences, Universiti Sains Malaysia, Kota Bharu, Malaysia
}

Irritable bowel syndrome (IBS), a common gastrointestinal disorder involving the gut-brain axis, and inflammatory bowel disease (IBD), a chronic relapsing inflammatory disorder, are both increasing in incidence and prevalence in Asia. Both have significant overlap in terms of symptoms, pathophysiology, and treatment, suggesting the possibility of IBS and IBD being a single disease entity albeit at opposite ends of the spectrum. We examined the similarities and differences in IBS and IBD, and offer new thoughts and approaches to the disease paradigm. (Intest Res 2016;14:297-304)

Key Words: Irritable bowel syndrome; Inflammatory bowel disease; Gut-brain axis; IBS-IBD overlap syndrome

\section{INTRODUCTION}

Irritable bowel syndrome (IBS) and IBD are two common chronic gastrointestinal (GI) disorders with unknown etiology and mechanisms. As our understanding improves, what were initially thought of as two separate and distinct GI disorders seem to have more in common, particularly at the extreme spectrum of both disorders-the prodromal phase of IBD and the late phase of IBS. This is augmented by the overlap of symptoms as well as the presence of colitis, raising the question of whether IBS and IBD are essentially on the same timeline-an evolution of the same disease.

\section{DILEMMA OF IBS-IBD}

IBS is characterized by a disordered gut-brain axis, but can

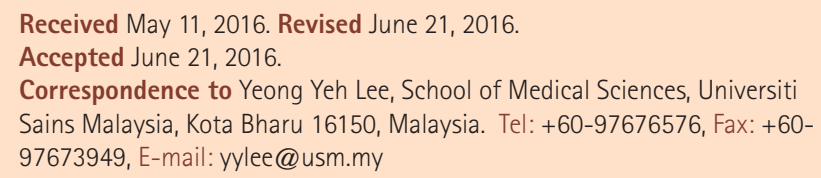

Financial support: None. Conflict of interest: None. develop following an enteric infection, and is associated with persistent immune activation that is a feature of IBD. Similarly, IBD, which encompasses CD and UC, is characterized by chronic relapsing inflammation and immune activation; however, recent evidence also points to altered gut microbiota and disturbed psychology, which are features of IBS, being important, both in the development and maintenance of disease.

The considerable overlap of symptoms and colitis raises the questions of whether IBS is a prodromal or mild subset of IBD, or whether IBD is pathologically related to the cause of IBS, or do they even represent the same pathophysiological spectrum of a disease. These claims are supported by the association and prevalence of IBS coexisting with IBD, especially in $\mathrm{CD}$, with $39 \%$ pooled prevalence and OR of 4.89 , even in remission. ${ }^{1}$

Indeed, there are a few studies correlating an increased risk of IBD among those with initial IBS symptoms. ${ }^{2-4}$

\footnotetext{
๑ Copyright 2016. Korean Association for the Study of Intestinal Diseases. All rights reserved.

This is an Open Access article distributed under the terms of the Creative Commons Attribution Non-Commercial License (http://creativecommons.org/licenses/by-nc/4.0)

which permits unrestricted non-commercial use, distribution, and reproduction in any medium, provided the original work is properly cited.
} 


\section{EPIDEMIOLOGY OF IBS AND IBD-BOTH ON THE RISE IN ASIA}

The incidence of $\mathrm{CD}$ in the world ranges from 5.0 to 10.7 per 100,000 person-years, while the incidence of UC ranges from 6.3 to 24.3 per 100,000 person-years. The marked variations are due to geographical localities, with Asia tending to show the lowest incidence rate, as compared to predominantly UC in Europe, and CD in North America. ${ }^{5}$ Even in Asia, with its large geographical area, there is variation in the annual incidence rate from 0.1 to 6.3 per 100,000 population for UC and 0.04 to 5.0 per 100,000 population for $\mathrm{CD}^{5}$

Gender differences were reportedly equal in large population-based studies, although some contested a higher male preponderance for IBD in Asia. ${ }^{5,6}$ The highest incidence ages of diagnosis were recorded in the second to fourth decades, therefore implicating the most productive age group, with socioeconomic impact in terms of hours off work and impaired productivity.

IBS in Asia shows a prevalence rate of $2.9 \%$ to $15.6 \%$, with no predilection for the traditionally female gender. ${ }^{7-9}$ The prevalence rate is highly dependent on the utilization of Manning or Rome-based criteria, and to a lesser extent on the geographical distribution. Age distribution still involves younger individuals in their early 20 's, comparable to Western studies.

However, for both IBS and IBD, the prevalence and annual incidence has shown a consistently increasing trend in Asia; which is in keeping with a worldwide trend.

Several studies from Asia spanning from 1986 to 2006 had shown increasing prevalence of IBD, ranging from 1.3 to 7.6 in the 1990 's to 6.3 to 30.9 per 100,000 in the new millennium. ${ }^{10-14}$ In contrast, IBS is more variable, but the general trend has been on the increase, especially in affluent cities such as Singapore and Tokyo, while some reports indicate a common syndrome affecting both rural and urban populations. ${ }^{15-18}$

\section{SIMILARITIES OF IBS AND IBD}

Apart from similarities in symptoms and signs, there are several other pathophysiological similarities between IBD and IBS. These can be broadly categorized into four main components, including the brain-gut axis, genetic factors, microbiota, and the epithelial barrier, among others.

\section{Brain-Gut Axis}

It is a well-known fact that both IBD and more particularly IBS are predisposed to psychological comorbidities, with a cause and effect relationship. There is a bidirectional interaction between the central nervous system and the enteric nervous system, which in turn modulates the gut function.

There is evidence that depression and anxiety are more common in IBD patients, with the symptoms being more severe during active disease. ${ }^{2}$ A large Swiss IBD Cohort Study involving 2,007 patients showed that although anxiety is more prevalent compared to depression in IBD patients, depression has a more significant negative effect on IBD activity. ${ }^{19}$ Depression in itself predisposes to increased inflammation in response to stress, by releasing a higher amount of pro-inflammatory cytokines such as interleukin 6 (IL-6), compared to normal controls, as proven in human studies and animal models. ${ }^{20,21}$ Interestingly, a bidirectional effect has also been shown, in which the IBD course is also worse in patients who are depressed. ${ }^{22-24}$

In IBS, it has been reported that $50 \%$ to $90 \%$ of patients have or had at some point one or more common psychiatric condition, including major depressive disorder, generalized anxiety disorder, social phobia, somatization disorder, or posttraumatic stress disorder. ${ }^{2,25}$ Our own limited data suggest that these psychological comorbidities in IBS are often nonserious. ${ }^{9}$ The limbic system is believed to be responsible by causing a surge in adrenocorticotrophic hormone and cortisol, and mediators such as IL-6 and IL-8 initiate a response in the enteric nervous system, resulting in symptoms of abdominal pain and diarrhea, which are typical of IBS. ${ }^{20,26}$ A more prominent activation of the dorsolateral prefrontal cortex area, which controls emotional and autonomic responses, has been shown to be increased in IBS patients, compared to control patients. ${ }^{27}$

\section{Genetic Factors}

The Tumor Necrosis Factor (Ligand)-Superfamily Member 15, also known as the TNF-SF15 gene, is known to be associated with CD and also primary biliary cirrhosis. The expression of this protein subsequently acts as an autocrine factor inducing apoptosis, and also inhibits endothelial cell proliferation, resulting in inflammation.

Several studies from the USA, Sweden, and more recently the UK have also noted the association of TNF-SF15 polymorphism with increased risk of IBS. ${ }^{28,29}$ This suggests a possible common pathway for both IBD and IBS through 
immune activation in both of these diseases.

Familial occurrence is common in both diseases, which highlights the possibility of shared genetic transmission. TNF-SF15 polymorphism may pave the way for identification of a precursor or trigger, whereas multi-gene analysis, such as the von Stein et al. ${ }^{30}$ seven gene model, may be utilized to differentiate between IBS and IBD.

\section{Microbiota}

Dysbiosis (abnormal gut microbiota) has been linked with several diseases that include IBD and IBS. A recent study evaluating a dysbiosis index algorithm detected dysbiosis in $70 \%$ of treatment-naïve IBD patients and $73 \%$ of IBS patients, in comparison with only $16 \%$ of healthy subjects. ${ }^{31}$

Alterations in gut microbiota have been observed in IBS patients, ${ }^{32,33}$ and are also seen in post-infectious IBS, which in turn is postulated to be a trigger for IBD ${ }^{33-36}$ Fluorescent insitu hybridization studies have detected increased bacterial presence in the mucus layer of IBD and IBS patients. Commensal organisms in IBD and IBS patients are also inherently different when compared to healthy subjects. ${ }^{34}$ Dysbiosis involving Faecalibacterium prausnitzii was noted to occur in a CD population in Europe, strengthening the argument for alterations of the intestinal microbiota as a cause of IBD. ${ }^{37,38}$

\section{Impaired Epithelial Barrier}

Increased gut permeability, which therefore increases susceptibility to injurious agents, has been suggested to precede clinical CD. ${ }^{39}$ Stress exacerbates IBD, and has been shown to cause an increase in activation of gut mast cells, which subsequently increases gut permeability. ${ }^{34,40}$

Similarly in IBS, elevation in miRNA-29a has been noted. This plays a role in down-regulating glutamine synthetase, which causes increased gut permeability, ${ }^{41}$ in a manner similar to IBD-related increases in permeability and subsequent injury.

Increased intestinal permeability due to changes at the cellular levels has been attributed to changes in transient receptor potential vanilloid receptor 1 , protein zonulin 1 , and $\alpha$-catenin, and has indeed been implicated in both IBS and also IBD presenting with IBS symptoms. ${ }^{18,42,43}$

Bacterial gastroenteritis as opposed to viral gastroenteritis also predisposes to greater permeability disturbances, and is associated with increased postinfectious IBS.

This common endpoint of increased gut permeability is currently the subject of intense studies worldwide.

\section{DIFFERENCES BETWEEN IBS AND IBD}

Notable differences were also seen between IBD and IBS, although there are arguments that these could be considered similarities. These include the following:

\section{Fecal Calprotectin}

The advent of fecal calprotectin has revolutionized noninvasive testing in IBD. High calprotectin levels are almost always due to ongoing inflammation related to chronic IBD. Keohane et al. ${ }^{44}$ found that IBD in remission with associated IBS exhibited greater fecal calprotectin levels than IBD in remission alone. This suggests that despite IBD being in remission, occult inflammation continues in the presence of IBS. In contrast, IBS is likely to have normal to low levels of calprotectin unless it is associated with a low degree of inflammation, as in postinfectious IBS. ${ }^{45}$

It has been proposed that a level below $40 \mu \mathrm{g} / \mathrm{g}$ is an indicator of no inflammation, whereas a level above $100 \mu \mathrm{g} /$ $\mathrm{g}$ indicates significant inflammation, suggesting IBD. However, at the in-between level of 40 to $100 \mu \mathrm{g} / \mathrm{g}$, it is uncertain whether this indicates a low level of IBD or IBS, or pre-IBD IBS. ${ }^{46}$ In comparison to other biomarkers, including high sensitivity CRP, lactoferrin, tumor necrosis factor (TNF)- $\alpha$, nitric oxide, and intraepithelial lymphocytes, fecal calprotectin has helped to identify active IBD patients, but not such that it has proved to be a good positive or negative predictor, as evident by being normal in IBS. ${ }^{44,47,48}$ These other biomarkers, however, may be more useful as part of a diagnostic workup in combination with calprotectin. ${ }^{49}$

\section{Degree of Inflammation}

In IBD, mucosal inflammation is usually ongoing and slow to resolve, even in clinically asymptomatic patients. IBD in remission still exhibits a higher level of TNF- $\alpha$ and intraepithelial lymphocytes compared to IBS patients. ${ }^{50}$ In contrast, IBS patients tend to exhibit low grade, variable, or even absent mucosal inflammation. ${ }^{2}$

\section{Symptoms versus Inflammation Mismatch}

Inherently, IBD is an organic disease, as evidenced by mucosal inflammation, whereas IBS lies more in the spectrum of a functional disorder, with no evidence of organic disease. IBS symptoms are nonspecific, and may precede diagnosis of both IBS and IBD by many years. Lack of mucosal inflam- 
mation results in a mismatch compared to the severity of the reported symptoms.

In IBD, mucosal inflammation is characteristic, but the symptoms do not necessarily correlate with endoscopic findings. ${ }^{51}$

\section{Visceral Hypersensitivity}

The gut viscera are controlled by a complex, incompletely understood interaction between the enteric nervous system, the vagal and spinal primary afferents, and both small and large myelinated and unmyelinated fibers that control motility and peristalsis. The interaction of neuroimmune and intestinal epithelial cells may prove to be a protective barrier in health but has also been implicated as the likely cause of GI pathology. In addition, there are persistent increases in mast cells, vasoactive intestinal peptide, and substance P, among many other receptors, which again either maintains or is a causative agent of GI disease.

As proven by persistent pain despite minimal inflammation and the response to centrally-acting agents, visceral hypersensitivity is the likely explanation for the symptoms and brain responses in IBS.

However, in IBD, the hallmark of the disease is mucosal inflammatory change that correlates with disease severity, and is the target of healing treatment. Visceral hypersensitivity is more apparent in IBD patients in remission, further strengthening the argument for IBS as a pre-IBD state.

\section{IBS-IBD DISEASE PARADIGMS}

There is growing evidence that IBS or IBS-like symptoms are a prodrome before the formal diagnosis of IBD. It has also been documented that IBS symptoms occur in IBD patients in remission, particularly in cases of $\mathrm{CD}$.

Over the years, several disease progression paradigms have been proposed (Fig. 1). Initially, after an episode of contaminated municipal water supply, it was proven that postinfectious IBS predates the formal diagnosis of IBS. ${ }^{52}$ In 2012, Porter et al. ${ }^{3}$ added to that work with the suggestion that post-infectious IBS is followed by IBS, and subsequently followed by active IBD. Berrill et al. ${ }^{53}$ suggested that IBS is an early part of a disease spectrum that subsequently leads to IBD, and progresses towards "subclinical IBD," in which case, mucosal healing might not be the endpoint in therapy. Stanisic and Quigley ${ }^{51}$ instead proposed "irritable IBD" as the unifying model of IBS symptoms in IBD in remission.

Our proposal that IBS and IBD comprise a single disease paradigm is not new, although variation exists as to what happens in-between the two conditions. Moreover, IBS is a disorder with a very broad spectrum, and immune activation has been found in only a fraction of cases. Thus, the association of IBS with IBD may be confined to this fraction of IBS. Indeed, further research is needed to support this idea. ${ }^{3,18,54,55}$

The initial or prodromal insult is enteric infection, resulting in postinfectious IBS, which is followed by a period of IBSlike symptoms without obvious colonic inflammation. We further propose an "early or pre-IBD" period at which there is a low level or grade of colonic inflammation occurring in IBS. This then leads to active IBD, followed by subclinical IBD with ongoing low-grade inflammation, although it is possible that irritable IBD occurs when the inflammation burns out.

This low-grade inflammation during the early pre-IBD period is suggested by studies showing that fecal calprotectin remains positive in one-third of IBS patients; this indicates that inflammation, along with further insults such as infections or stress, may inadvertently trigger IBD, followed by the extreme end of the spectrum, which is the proposed subclinical IBD (Fig. 1). Other studies showed that microscopic inflammation was found in up to $14.9 \%$ of cases in diarrheapredominant IBS, supporting the claim of low-level ongoing inflammation prior to the diagnosis of IBD. ${ }^{56}$ Indeed, patients

$$
\text { PI-IBS }
$$$$
\text { PI-IBS }
$$$$
\text { IBS }
$$$$
\text { IBD }
$$$$
\text { IBS }
$$

Marshall et al. (2010) ${ }^{66}$

Porter et al. (2012)

Berrill et al. $(2013)^{53}$

\section{IBS}

$\mathrm{IBD}$

IIBS

Stanisic and Quigley (2014)

PI-IBS

IBS

IBD

One disease paradigm
Fig. 1. Disease paradigms in IBS-IBD. Existing paradigms and our proposed one disease paradigm are shown here. These paradigms illustrate the evolution of concepts in IBS-IBD overlap syndrome. PI-IBS, postinflammatory IBS; IIBS, irritable IBS. 
with IBS are 15 times more likely to develop IBD compared to those with no IBS-like symptoms. ${ }^{3}$

The question is what may have caused the ongoing lowgrade inflammation in the early or pre-IBD period? We believe that altered gut-brain axis and disturbed psychology associated with IBS can perpetuate and sustain the low-grade inflammation. Likewise, new triggers including new enteric infection in the form of small intestinal bacterial overgrowth (SIBO) or intestinal dysbiosis, that is often unsuspected in IBS or IBD, may have sustained the low-grade inflammation. Bloating is a common symptom present in both disorders, and SIBO is a cause of bloating that can be excluded easily through hydrogen breath testing. ${ }^{57,58}$

\section{WHAT TO OFFER THESE PATIENTS?}

The main challenge has always been to make a definitive diagnosis, but overlap between IBS and IBD can pose a problem. A colonoscopy with mucosal histopathological studies and/or Rome questionnaires may not be adequate to separate the two. Management consideration is shown in Fig. 2.

With fecal calprotectin (or other stool markers, e.g., lactoferrin), it is potentially easier to distinguish between IBD, IBS, or the proposed early or pre-IBD condition with its lowgrade inflammation. This can further be of use as a riskstratifying method to ensure these patients are followed up, thereby preventing or controlling active IBD.

Regulation of the gut microbiota as a potential trigger of IBS and IBD is also important. This therefore necessitates testing for SIBO or intestinal dysbiosis, and future strategies including the use of prebiotics, probiotics, or synbiotics are needed.

Often neglected but proven is the bidirectional relationship of anxiety and depression or other altered psychology states in IBD or IBS. Therefore, it is essential to have a holistic approach and to address such concerns in not only cases of active disease but for those in remission as well. ${ }^{59-62}$

\section{FUTURE RESEARCH}

Current research into IBS-IBD similarities has so far only scratched the surface. Further gene studies including NOD2 and IBD1-5 among others should be conducted to complement current information gleaned from the TNF-SF15 information we currently have.

Emerging gut microbiota research should be able to influence the management of IBS and IBD with utilization of pre/ probiotics and perhaps vaccination strategies.

Gut-brain axis studies involving hypnosis and psychotherapy are beginning to show promising results, prompting a more inclusive view and stressing the importance of a multidisciplinary approach. Several studies are currently underway to assess the effect of IBS drugs such as tricyclics, and IBD drugs such as mesalamine, when used interchangeably to treat the opposite disorders. In several studies completed so far, although the above drugs had no major impact in IBS patients, there were improvements in some subtypes of IBS, suggesting that these drugs may be useful in patients at a certain threshold or timeline in their evolution of the IBSIBD paradigm. ${ }^{63-65}$

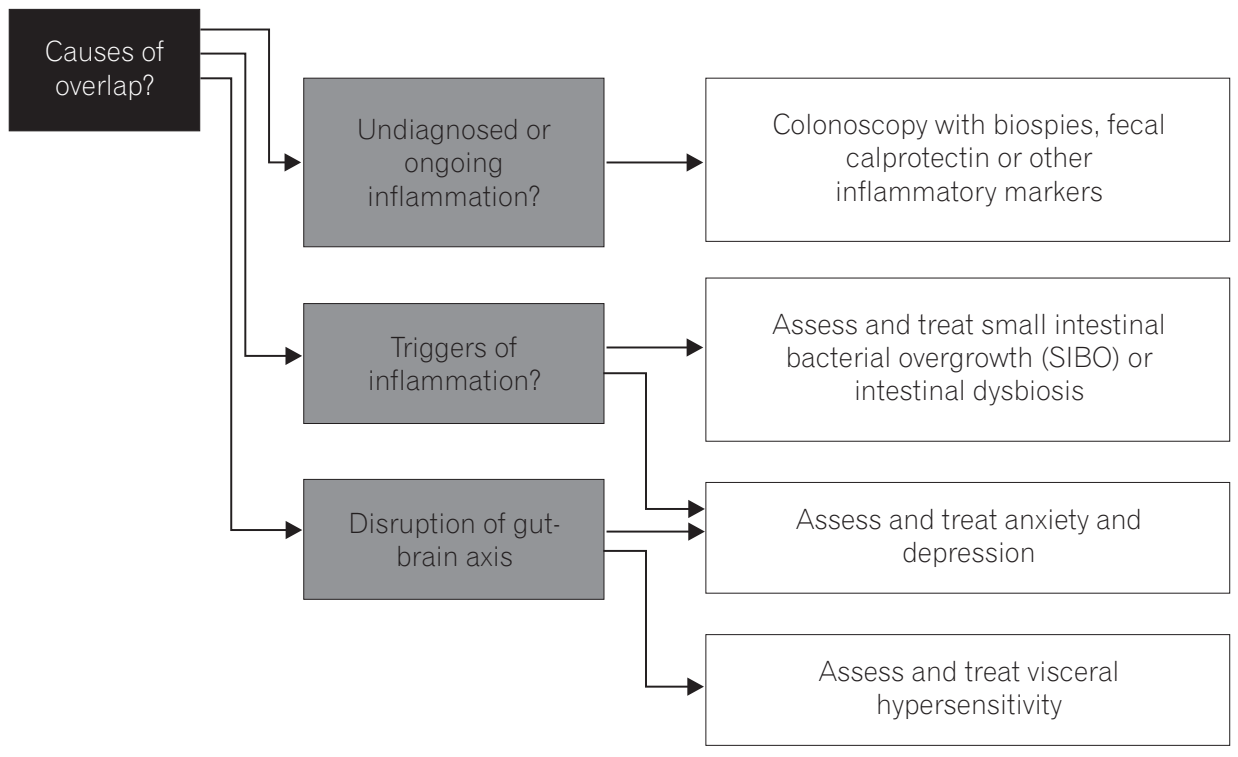

Fig. 2. Management consideration in IBS$\mathrm{IBD}$ overlap. When considering progression or overlap of IBS-IBD, it is important to exclude undiagnosed or ongoing inflammation, and thus the need for biomarkers including fecal calprotectin (or others, either existing or in development) and pathological assessment. Triggers for ongoing inflammation are also sought especially occult infection and psychological dysfunction which are often subtle and not noticed. It is also important to assess and treat other disorders of the gut-brain axis (including visceral hypersensitivity). 


\section{CONCLUSIONS}

Previously disputed, the idea of IBS and IBD being intimately interlinked seems to be gathering pace, backed by a litany of evidence and research developments. The disease paradigm may have to be altered to consider both IBS and IBD as belonging on the same timeline, but with differing presentation and outlook, allowing a more comprehensive management plan.

Ultimately, further research and studies into these particular areas may inadvertently lead to prevention strategies for IBS, thereby negating the subsequent consequences of IBD.

\section{REFERENCES}

1. Halpin SJ, Ford AC. Prevalence of symptoms meeting criteria for irritable bowel syndrome in inflammatory bowel disease: systematic review and meta-analysis. Am J Gastroenterol 2012;107:1474-1482.

2. García Rodríguez LA, Ruigómez A, Wallander MA, Johansson S, Olbe L. Detection of colorectal tumor and inflammatory bowel disease during follow-up of patients with initial diagnosis of irritable bowel syndrome. Scand J Gastroenterol 2000;35:306-311.

3. Porter CK, Cash BD, Pimentel M, Akinseye A, Riddle MS. Risk of inflammatory bowel disease following a diagnosis of irritable bowel syndrome. BMC Gastroenterol 2012;12:55.

4. Porter CK, Tribble DR, Aliaga PA, Halvorson HA, Riddle MS. Infectious gastroenteritis and risk of developing inflammatory bowel disease. Gastroenterology 2008;135:781-786.

5. Molodecky NA, Soon IS, Rabi DM, et al. Increasing incidence and prevalence of the inflammatory bowel diseases with time, based on systematic review. Gastroenterology 2012;142:46-54. e42.

6. Park SJ, Kim WH, Cheon JH. Clinical characteristics and treatment of inflammatory bowel disease: a comparison of Eastern and Western perspectives. World J Gastroenterol 2014;20:11525-11537.

7. Lee OY. Prevalence and risk factors of irritable bowel syndrome in Asia. J Neurogastroenterol Motil 2010;16:5-7.

8. Gwee KA, Bak YT, Ghoshal UC, et al. Asian consensus on irritable bowel syndrome. J Gastroenterol Hepatol 2010;25:11891205.

9. Lee YY, Waid A, Tan HJ, Chua AS, Whitehead WE. Rome III survey of irritable bowel syndrome among ethnic Malays. World J Gastroenterol 2012;18:6475-6480.

10. Yao T, Matsui T, Hiwatashi N. Crohn's disease in Japan: diagnostic criteria and epidemiology. Dis Colon Rectum 2000;43(10 Suppl):S85-S93.
11. Yang SK, Yun S, Kim JH, et al. Epidemiology of inflammatory bowel disease in the Songpa-Kangdong district, Seoul, Korea, 1986-2005: a KASID study. Inflamm Bowel Dis 2008;14:542549.

12. Thia KT, Loftus EV Jr, Sandborn WJ, Yang SK. An update on the epidemiology of inflammatory bowel disease in Asia. Am J Gastroenterol 2008;103:3167-3182.

13. Lok KH, Hung HG, Ng CH, et al. Epidemiology and clinical characteristics of ulcerative colitis in Chinese population: experience from a single center in Hong Kong. J Gastroenterol Hepatol 2008;23:406-410.

14. Prideaux L, Kamm MA, De Cruz PP, Chan FK, Ng SC. Inflammatory bowel disease in Asia: a systematic review. J Gastroenterol Hepatol 2012;27:1266-1280.

15. Zhu X, Chen W, Zhu X, Shen Y. A cross-sectional study of risk factors for irritable bowel syndrome in children 8-13 years of age in Suzhou, China. Gastroenterol Res Pract 2014;2014:198461.

16. Gwee KA. Irritable bowel syndrome in developing countries: a disorder of civilization or colonization? Neurogastroenterol Motil 2005;17:317-324.

17. Gwee KA, Lu CL, Ghoshal UC. Epidemiology of irritable bowel syndrome in Asia: something old, something new, something borrowed. J Gastroenterol Hepatol 2009;24:1601-1607.

18. Quigley EM. Overlapping irritable bowel syndrome and inflammatory bowel disease: less to this than meets the eye? Therap Adv Gastroenterol 2016;9:199-212.

19. Mikocka-Walus A, Pittet V, Rossel JB, von Känel R; Swiss IBD Cohort Study Group. Symptoms of depression and anxiety are independently associated with clinical recurrence of inflammatory bowel disease. Clin Gastroenterol Hepatol 2016;14:829835.el.

20. Fagundes CP, Glaser R, Hwang BS, Malarkey WB, Kiecolt-Glaser JK. Depressive symptoms enhance stress-induced inflammatory responses. Brain Behav Immun 2013;31:172-176.

21. Johnson JD, O'Connor KA, Deak T, Stark M, Watkins LR, Maier SF. Prior stressor exposure sensitizes LPS-induced cytokine production. Brain Behav Immun 2002;16:461-476.

22. Salazar G. Depression and IBD. J Pediatr Gastroenterol Nutr 2014;58:543-544.

23. Mikocka-Walus AA, Turnbull DA, Moulding NT, Wilson IG, Andrews JM, Holtmann GJ. Controversies surrounding the comorbidity of depression and anxiety in inflammatory bowel disease patients: a literature review. Inflamm Bowel Dis 2007;13:225234.

24. Graff LA, Walker JR, Bernstein CN. Depression and anxiety in inflammatory bowel disease: a review of comorbidity and management. Inflamm Bowel Dis 2009;15:1105-1118. 
25. Lydiard RB. Irritable bowel syndrome, anxiety, and depression: what are the links? J Clin Psychiatry 2001;62 Suppl 8:38-45.

26. Padhy SK, Sahoo S, Mahajan S, Sinha SK. Irritable bowel syndrome: is it "irritable brain" or "irritable bowel"? J Neurosci Rural Pract 2015;6:568-577.

27. Jones MP, Dilley JB, Drossman D, Crowell MD. Brain-gut connections in functional GI disorders: anatomic and physiologic relationships. Neurogastroenterol Motil 2006;18:91-103.

28. Zucchelli M, Camilleri M, Andreasson AN, et al. Association of TNFSF15 polymorphism with irritable bowel syndrome. Gut 2011;60:1671-1677.

29. Swan C, Duroudier NP, Campbell E, et al. Identifying and testing candidate genetic polymorphisms in the irritable bowel syndrome (IBS): association with TNFSF15 and TNF-alpha. Gut 2013;62:985-994.

30. von Stein P, Lofberg R, Kuznetsov NV, et al. Multigene analysis can discriminate between ulcerative colitis, Crohn's disease, and irritable bowel syndrome. Gastroenterology 2008;134:18691881

31. Casén C, Vebø HC, Sekelja M, et al. Deviations in human gut microbiota: a novel diagnostic test for determining dysbiosis in patients with IBS or IBD. Aliment Pharmacol Ther 2015;42:7183.

32. Quigley EM. Bacterial flora in irritable bowel syndrome: role in pathophysiology, implications for management. J Dig Dis 2007;8:2-7.

33. Lee YY, Rao SS. Post-infectious irritable bowel syndrome. Biotascope 2015;2:10-16.

34. Spiller R, Lam C. The shifting interface between IBS and IBD. Curr Opin Pharmacol 2011;11:586-592.

35. Spiller R, Campbell E. Post-infectious irritable bowel syndrome. Curr Opin Gastroenterol 2006;22:13-7.

36. Rhodes DY, Wallace M. Post-infectious irritable bowel syndrome. Curr Gastroenterol Rep 2006;8:327-332.

37. Joossens M, Huys G, Cnockaert M, et al. Dysbiosis of the faecal microbiota in patients with Crohn's disease and their unaffected relatives. Gut 2011;60:631-637.

38. Sokol H, Seksik P, Furet JP, et al. Low counts of Faecalibacterium prausnitzii in colitis microbiota. Inflamm Bowel Dis 2009;15:1183-1189.

39. Hedin CR, Stagg AJ, Whelan K, Lindsay JO. Family studies in Crohn's disease: new horizons in understanding disease pathogenesis, risk and prevention. Gut 2012;61:311-318.

40. Bernstein CN, Singh S, Graff LA, Walker JR, Miller N, Cheang M. A prospective population-based study of triggers of symptomatic flares in IBD. Am J Gastroenterol 2010;105:1994-2002.
41. Zhou Q, Souba WW, Croce CM, Verne GN. MicroRNA-29a regulates intestinal membrane permeability in patients with irritable bowel syndrome. Gut 2010;59:775-784.

42. Akbar A, Walters JR, Ghosh S. Review article: visceral hypersensitivity in irritable bowel syndrome: molecular mechanisms and therapeutic agents. Aliment Pharmacol Ther 2009;30:423435 .

43. Keszthelyi D, Jonkers DM, Hamer HM, Masclee AA. Letter: the role of sub-clinical inflammation and TRPV1 in the development of IBS-like symptoms in ulcerative colitis in remission. Aliment Pharmacol Ther 2013;38:560-561.

44. Keohane J, O'Mahony C, O'Mahony L, O’Mahony S, Quigley EM, Shanahan F. Irritable bowel syndrome-type symptoms in patients with inflammatory bowel disease: a real association or reflection of occult inflammation? Am J Gastroenterol 2010;105:1789-1794.

45. Melchior CA, Aubry T, Gourcerol G, Leroi AM, Ducrotté P. Fecal calprotectin levels in IBS patients: results from prospective study. United European Gastroenterol J 2013;1(Suppl 1):A68.

46. Jelsness-Jørgensen LP, Bernklev T, Moum B. Calprotectin is a useful tool in distinguishing coexisting irritable bowel-like symptoms from that of occult inflammation among inflammatory bowel disease patients in remission. Gastroenterol Res Pract 2013;2013:620707.

47. Hod K, Ringel-Kulka T, Martin CF, Maharshak N, Ringel Y. Highsensitive C-reactive protein as a marker for inflammation in irritable bowel syndrome. J Clin Gastroenterol 2016;50:227-232.

48. Zhou XL, Xu W, Tang XX, et al. Fecal lactoferrin in discriminating inflammatory bowel disease from irritable bowel syndrome: a diagnostic meta-analysis. BMC Gastroenterol 2014;14:121.

49. Menees SB, Powell C, Kurlander J, Goel A, Chey WD. A meta-analysis of the utility of C-reactive protein, erythrocyte sedimentation rate, fecal calprotectin, and fecal lactoferrin to exclude inflammatory bowel disease in adults with IBS. Am J Gastroenterol 2015;110:444-454.

50. Vivinus-Nébot M, Frin-Mathy G, Bzioueche H, et al. Functional bowel symptoms in quiescent inflammatory bowel diseases: role of epithelial barrier disruption and low-grade inflammation. Gut 2014;63:744-752.

51. Stanisic V, Quigley EM. The overlap between IBS and IBD: what is it and what does it mean? Expert Rev Gastroenterol Hepatol 2014;8:139-145.

52. Villani AC, Lemire M, Thabane M, et al. Genetic risk factors for post-infectious irritable bowel syndrome following a waterborne outbreak of gastroenteritis. Gastroenterology 2010;138:1502-1513. 
53. Berrill JW, Green JT, Hood K, Campbell AK. Symptoms of irritable bowel syndrome in patients with inflammatory bowel disease: examining the role of sub-clinical inflammation and the impact on clinical assessment of disease activity. Aliment Pharmacol Ther 2013;38:44-51.

54. Bercik P, Verdu EF, Collins SM. Is irritable bowel syndrome a low-grade inflammatory bowel disease? Gastroenterol Clin North Am 2005;34:235-245.

55. Quigley EM. Irritable bowel syndrome and inflammatory bowel disease: interrelated diseases? Chin J Dig Dis 2005;6:122-132.

56. Hilmi I, Hartono JL, Pailoor J, Mahadeva S, Goh KL. Low prevalence of 'classical' microscopic colitis but evidence of microscopic inflammation in Asian irritable bowel syndrome patients with diarrhoea. BMC Gastroenterol 2013;13:80.

57. Erdogan A, Rao SS, Gulley D, Jacobs C, Lee YY, Badger C. Small intestinal bacterial overgrowth: duodenal aspiration vs glucose breath test. Neurogastroenterol Motil 2015;27:481-489.

58. Rao SS, Lee YY. Approach to the patient with gas and bloating. In: Podolsky DK, Camilleri M, Fitz JG, Kalloo AN, Shanahan F, Wang TC, eds. Yamada's textbook of gastroenterology. Oxford: John Wiley \& Sons, 2015: 723-734.

59. Keefer L, Taft TH, Kiebles JL, Martinovich Z, Barrett TA, Palsson OS. Gut-directed hypnotherapy significantly augments clinical remission in quiescent ulcerative colitis. Aliment Pharmacol Ther 2013;38:761-771.
60. Rutten JM, Vlieger AM, Frankenhuis C, et al. Gut-directed hypnotherapy in children with irritable bowel syndrome or functional abdominal pain (syndrome): a randomized controlled trial on self exercises at home using CD versus individual therapy by qualified therapists. BMC Pediatr 2014;14:140.

61. Triantafillidis JK, Merikas E, Gikas A. Psychological factors and stress in inflammatory bowel disease. Expert Rev Gastroenterol Hepatol 2013;7:225-238.

62. Triantafillidis JK, Vagianos K, Rontos I. Psychotic reaction as a cardinal first clinical manifestation in a patient with Crohn's disease. J Crohns Colitis 2013;7:e76-e77. doi: 10.1016/ j.crohns.2012.07.003.

63. Barbara G, Cremon C, Annese V, et al. Randomised controlled trial of mesalazine in IBS. Gut 2016;65:82-90.

64. Iskandar HN, Cassell B, Kanuri N, et al. Tricyclic antidepressants for management of residual symptoms in inflammatory bowel disease. J Clin Gastroenterol 2014;48:423-429.

65. Lam C, Tan W, Leighton M, et al. A mechanistic multicentre, parallel group, randomised placebo-controlled trial of mesalazine for the treatment of IBS with diarrhoea (IBS-D). Gut 2016;65:91-99.

66. Marshall JK, Thabane M, Garg AX, et al. Eight year prognosis of postinfectious irritable bowel syndrome following waterborne bacterial dysentery. Gut 2010;59:605-611. 